\title{
XEDS and EELS in the TEM at Atmospheric Pressure and High Temperature.
}

Eric Prestat ${ }^{1}$, Matthew Smith ${ }^{1}$, Arne Janssen ${ }^{1}$, Thomas J. A. Slater ${ }^{1}$, Pedro H. C. Camargo ${ }^{2}$, Matthew A. Kulzick $^{3}$, M. Grace Burke ${ }^{1}$, Sarah J. Haigh ${ }^{1}$ and Nestor J. Zaluzec ${ }^{1,4}$

1. School of Materials, University of Manchester, Manchester, M13 9PL, UK

2. Instituto de Química, Universidade de São Paulo, São Paulo 05508-000, Brazil

3. BP Corporate Research Center, Naperville, IL 60563, USA

4. Electron Microscopy Center, NST Division, Argonne National Laboratory, Argonne, USA.

Recent progress with environmental cell and microscope design has enabled in situ imaging studies within gaseous environments inside the (scanning) transmission electron microscope ((S)TEM) to become increasingly routine [1-2]. In contrast, complementary elemental information is more challenging to obtain in situ. Electron energy loss spectroscopy (EELS) has been reported inside an environmental TEM [3,4], although only at modest pressure (below 30 mbar). Closed-cell design specimen holders, in which the specimen and the gaseous environment are sealed from the high vacuum of the TEM by two SiN windows, allow much higher pressures to be reached (up to $\sim 1$ bar). However, these environmental cells have two major drawbacks which limit their analytical capabilities: 1) X-ray energy dispersive spectroscopy (XEDS) is challenging, as the walls of the cell generally shadow the detectors, preventing the collection of characteristic X-rays; 2) EELS is challenging as the two $50 \mathrm{~nm}$ thick SiN windows cause multiple scattering, which limits signal-to-background ratio in the core-loss EEL spectra and thus significantly degrades performance [5].

Recent improvement in the detection efficiency of both XEDS and EELS allows spectrum images (SIs) to be acquired at high speed and low total electron dose. In this presentation, we demonstrate the improved analytical performance of a closed-cell holder and its application to study alloying and segregation phenomena in PdAg nanoparticles during an in situ experiment. We demonstrate that the use of a XEDS compatible Protochips Atmosphere holder in a FEI Titan ChemiSTEM allows elemental mapping to be performed at high spatial resolution, ambient pressure and high temperature using XEDS.

Figure 1 compares XEDS and EELS maps acquired at $820 \mathrm{mbar} \mathrm{H}_{2}$ and $200^{\circ} \mathrm{C}$. The specimen holder was tilted to $25^{\circ}$ and only two of the four Super-X XEDS detectors were used. The probe current was set to $600 \mathrm{pA}$ to perform elemental mapping with a pixel time of $20 \mathrm{~ms}$ and a pixel size of $5 \AA$. The EELS "background" relative thickness $\mathrm{t} / \lambda$ is $\sim 0.8$, due to the two $50 \mathrm{~nm}$ thick SiN windows - measured with a $200 \mathrm{kV}$ incident electron beam, $81 \mathrm{mrad}$ EELS collection angle and $\sim 15 \mathrm{um}$ gas channel height at 1 bar $\mathrm{H}_{2}$. This makes EELS analysis increasingly problematic for specimens with thicknesses greater than $\sim 50$ $\mathrm{nm}$, while XEDS is still effective for much thicker specimens. As the nanoparticles used here have diameters of $\sim 30 \mathrm{~nm}$ the Pd and Ag EELS elemental maps in Figure 1 show slightly better signal-tonoise ratio than the corresponding XEDS maps. However, even for these small nanoparticles the background increases significantly on the particles themselves leading to a decrease of the signal-tobackground ratio of the $\mathrm{Si}$ edge (figure $1 \mathrm{j}$ ). Figure 2 shows XEDS maps acquired at $910 \mathrm{mbar} 5 \% \mathrm{O}_{2} / \mathrm{Ar}$ and $350^{\circ} \mathrm{C}$. The $\mathrm{PdAg}$ nanoparticles change morphology and begin alloying at temperatures above $300^{\circ} \mathrm{C}$. To study this it is essential to perform spectrum imaging, as the very similar atomic number of $\mathrm{Pd}$ and $\mathrm{Ag}$ (46 and 47, respectively) prevents identification using STEM-HAADF imaging alone. Spectroscopic imaging requires higher total electron dose than standard imaging, therefore work is in progress to control beam-induced segregation. 


\section{References}

[1] S. Giorgio et al, Ultramicroscopy 106, (2006), p. 503

[2] J. F. Creemer et al, Ultramicroscopy 108, (2008), p. 993

[3] S. Chenna and P. Crozier, Microsc. Microanal. 17 (Suppl 2), (2011), p. 476

[4] P. Crozier and S. Chenna, Ultramicroscopy 111, (2011), p. 177

[5] N. J. Zaluzec et al, Microsc. Microanal. 20, (2014), p. 323

[6] Research supported by EPSRC Grants \#EP/G035954/1 and EP/J021172/1, the DTR Agency Grant HDTRA1-12-1-003, the BP Innovation Fund and ICAM project at Manchester U.S. DoE, Office of Science, Contract No. DE-AC02-06CH11357 at Argonne National Laboratory

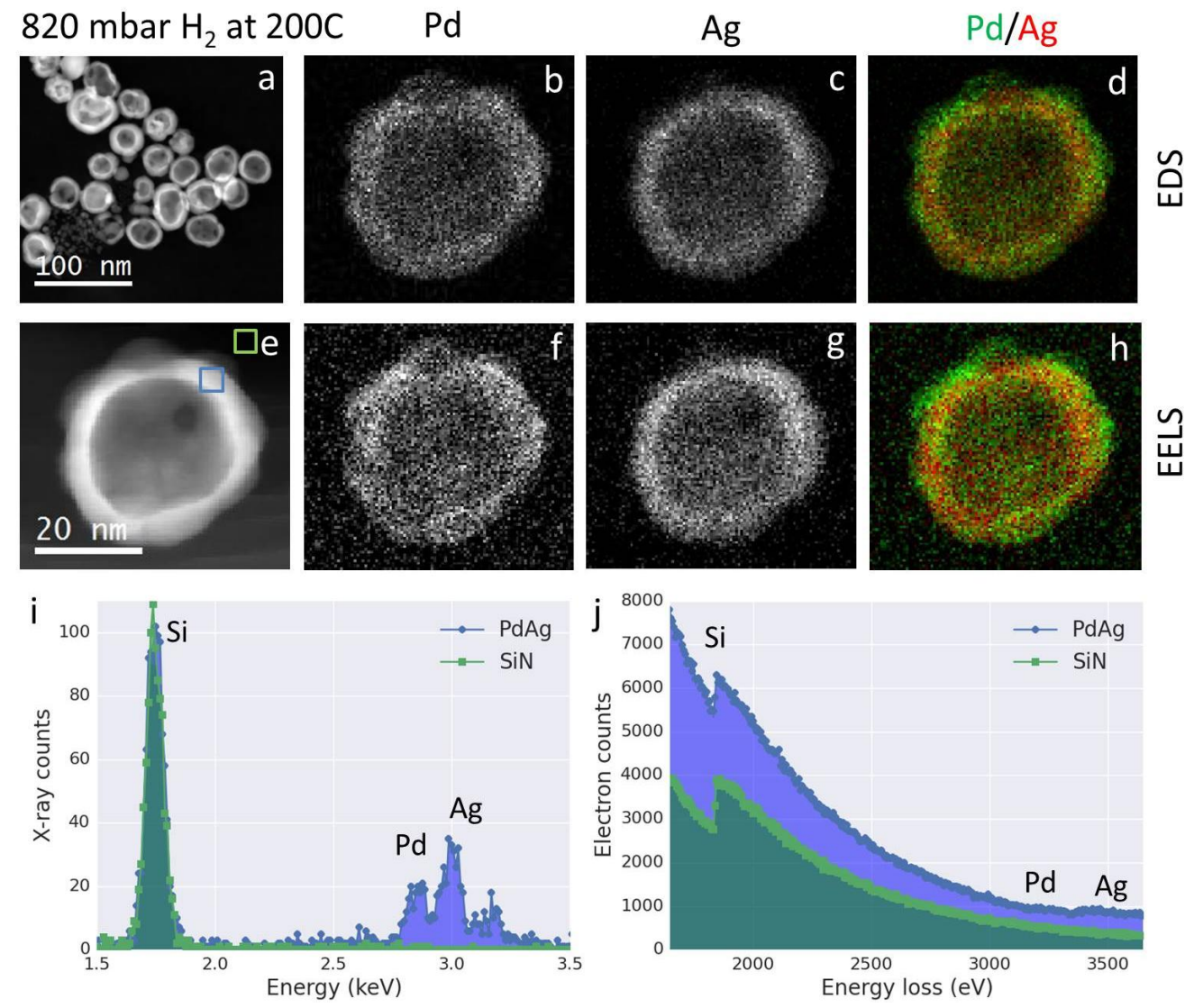

Figure 1. a) Representative STEM-HAADF image showing PdAg nanoparticles in a $820 \mathrm{mbar} H 2$ at $200^{\circ} \mathrm{C}$. b) $\mathrm{Pd}$, c) $\mathrm{Ag}$ and d) $\mathrm{Pd} / \mathrm{Ag}$ composite maps obtained using XEDS. e) STEM-HAADF image acquired simultaneously than the SIs. f) $\mathrm{Pd}, \mathrm{g}$ ) $\mathrm{Ag}$ and h) $\mathrm{Pd} / \mathrm{Ag}$ composite maps obtained using EELS. i) XED and j) EEL spectra of the positions marked in e). All spectra are integrated over 25 pixels.
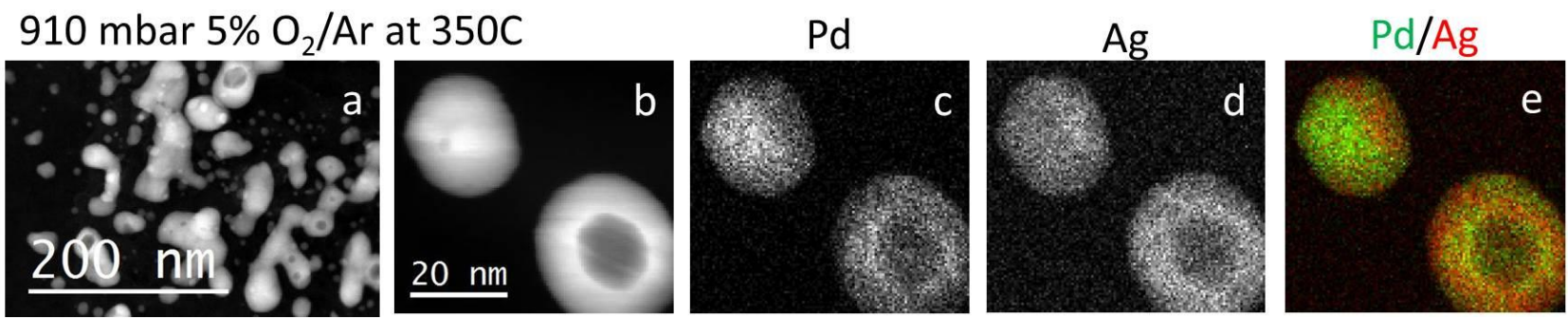

Figure 2. a) Representative STEM-HAADF image of the PdAg nanoparticles at 910 mbar and $350^{\circ} \mathrm{C}$ be) Spectrum imaging of elemental distribution b) STEM-HAADF c) Pd, d) Ag and e) Pd/Ag XEDS composite maps. 